\title{
Relatives of people with spinal cord injury: a qualitative study of caregivers' metamorphosis
}

\author{
Laura Juguera Rodriguez ${ }^{1}$ Manuel Pardo Rios ${ }^{1}$ - César Leal Costa $\mathbb{1}^{1} \cdot$ Matilde Castillo Hermoso ${ }^{2}$. \\ Nuria Perez Alonso ${ }^{1}$. Jose Luis Diaz Agea $\mathbb{D}^{1}$
}

Received: 15 June 2017 / Revised: 5 January 2018 / Accepted: 1 February 2018 / Published online: 21 March 2018

(C) International Spinal Cord Society 2018

\begin{abstract}
Study design Qualitative study.

Objective To detect the major challenges and needs reported by family member caregivers of people with spinal cord injury (SCI).

Setting Family member caregivers of people with SCI and expert professionals were evaluated. This study was conducted in Spain, and most of the participants attended the National Paraplegics Hospital of Toledo.

Methods We performed 25 semi-structured interviews. The data were analyzed from a phenomenological perspective using the Colaizzi method.

Results The metamorphosis of the caregiver is a complex personal and family-related process. Analysis of the adjustment phase of the caregiving role allowed us to describe three stages, patterns, and trends. Five basic needs were identified.

Conclusions People with SCI and their primary caregivers experienced changes in every sphere of their lives. Their most important needs were psychological support, social support, economic resources, information, training throughout the process of suffering, and the creation of informal groups of mutual aid.
\end{abstract}

\section{Introduction}

The occurrence of a spinal cord injury (SCI) implies a drastic change in the lives of the people who incur the injury as well as members of their immediate families. The consequences of this injury severely affect the capabilities and functions of individuals in their entirety [1]. SCI has an important effect within the family, causing a restructuring of family roles [2]. Family members must adopt the roles of caregivers and must become the protagonists of care, which changes their way of life, their planned objectives, and their personal relationships [3]. Taking on the role of caregiver can have several negative consequences, both physical and emotional; it can alter social and work relationships as well

Laura Juguera Rodriguez

ljuguera@ucam.edu

1 Department of Nursing, Catholic University of Murcia (UCAM), Murcia, Spain

2 National Hospital for Paraplegics, Toledo, Spain as cause dissatisfaction and emotional tension [4]. Studies report that caregivers even neglect their own health $[5,6]$.

In general, family members are unprepared for taking on the caregiving role as they face an unknown and unexpected situation. Furthermore, they must take responsibility for caring for the family member with SCI upon discharge from the hospital [5], which causes high rates of anxiety, depression, and stress $[3,4,7,8]$. During the rehabilitation period, the person with SCI and the caregiver must prepare for what could happen after being discharged. Support should be provided to clarify all doubts as well as to acquire the knowledge and skills needed to adapt to a new and possibly more stressful life. Social networks and access to rehabilitation centers to maintain contact with health professionals and to acquire knowledge are indispensable tools for avoiding the complications that arise from SCI and to avoid increasing the burden on the caregiver over the long term [9].

Few qualitative studies have revealed the gaps and needs of these caregivers during the rehabilitation period [10]. The extant literature has focused on quantitative studies of quality of life of caregivers at home, but they have not focused on how these caregivers might be helped during 
rehabilitation to provide them with an active role and involve them over this time period.

The objective of this study was to identify the major struggles, needs, and problems identified by the primary caregivers of people with SCI during the rehabilitation period and to contrast their perceptions with the opinions of expert professionals.

\section{Methods}

\section{Study design}

A qualitative phenomenological methodology was employed.

\section{Participants}

Participants included primary caregivers of people with SCI as well as health professional experts in SCI.

\section{Sample}

The sample was chosen from the National Association of Spinal Cord Injuries and Major Physical Handicaps (ASPAYM) of the Region of Murcia, the Virgen de la Arrixaca University Hospital (HUVA), and the National Paraplegics Hospital of Toledo between May 2013 and July 2015. The inclusion criteria were previous or current status as a primary caregiver of a person having a SCI or a healthcare professional with at least 2 years of experience in the care of people with SCI.

The fieldwork was complete when saturation of information was reached, with a final number of 25 volunteer informants (16 primary caregivers and 9 SCI experts). Saturation of information was reached when a phenomenon was interpreted or described in the same manner by most of the participants and no new information was identified.

\section{Data collection and analysis}

Semi-structured interviews were conducted, and the structure of the interview script was established based on the proposed objectives, using open-ended questions (Table 1). The same script structure was used for the skilled health professionals, although the formulation of the questions was modified.

Once all were recorded, they were transcribed, and an alphanumeric code was assigned to each interview. A discourse analysis was performed using the software program MAXQDA- $12^{\circ}$ and by following the Colaizzi method [11]. First, each transcript was read several times to obtain a general idea of the content. Then, significant fragments related to the phenomenon under study were extracted. The next step consisted of formulating the meaning of each fragment to later organize these fragments into themes, sets of themes, and categories. Simultaneously, the themes that emerged throughout the process of analysis of meaning were defined. After all of the emerging categories and themes were merged, a comprehensive description of the phenomenon of study was conducted. Once the extracted information was put into perspective and codified, matrices and relation models between the categories were created in order to obtain association patterns that allowed for a better understanding of the study subject. The results obtained from the expert researchers were reviewed to confirm that the descriptions extracted mirrored the perceptions, experiences, and needs of the caregivers of people with SCI.

The results were validated by returning the descriptions to the participants for review. The reliability of the data was ensured as the research team independently corroborated the transcription and interpretation of the results.

Table 1 Interview topics

Topics addressed during the interview

\begin{tabular}{lc}
\hline Caregivers & Professionals
\end{tabular}

(1) Needs during the rehabilitation period

(2) Deficiencies detected during the rehabilitation process

(3) What are your support pillars?

(4) Information received during the rehabilitation period

(5) Involvement in care during the rehabilitation period

(6) Training during the rehabilitation period, professionals involved

(7) Health professional response to demands
(1) What are the needs of the primary caregivers of the person with SCI during the rehabilitation period?

(2) In what way do you involve the caregiver in your daily activities?

(3) Do you think that shortcomings exist in the care provided?

(4) What information do you provide to caregivers, and how is this information delivered?

(5) In what ways can the caregiver acquire competencies?

(6) What do you think are the fundamental pillars of caregiver support? 


\section{Ethical considerations}

No fundamental human rights were violated, and ethical criteria were followed throughout the study. The participants signed informed consent documents. The confidentiality of the data was respected, and the privacy of the participants was assured. The UCAM and NHP ethics committee approved the research protocol, and the ethical principles of the Declaration of Helsinki were followed.

\section{Results}

The results obtained in this study are shown structured in sections for better comprehension. The three sections are: (a) description of context and background (b) the metamorphosis of the caregiver, and (c) the needs of the caregiver, detected by the primary caregiver since the SCI occurred. Table 2 shows the emerging categories and themes of this study. Below, we detail the results and the most relevant discourses of our research.

\section{Description of context and background}

Most of the hospitals in Spain refer patients with SCI to a specialized hospital to receive comprehensive rehabilitation treatment. The center the patients are usually referred to is the National Hospital of Paraplegics (NHP), a leading specialized hospital for the rehabilitation of patients with SCI. The average rehabilitation stay associated with SCI is 6-12 months, a long period during which the family must organize and assume new roles. The lengthy hospitalization creates a drastic change in everyone's life, unraveling the family's nucleus. Once the rehabilitation stage ends, the patient will be discharged to his or her home. At home, there is no professional medical assistance, so in most cases, one of the family members will have to take on the role of caregiver. Thus, one of the main objectives of the stay in the NHP is training a caregiver.

During the hospital stay, relatives can join the patient during the day in some activities of the rehabilitation process. As for the specific roles played by family caregivers during the period of rehabilitation, there was no standardized protocol found in the center. Greater or lesser involvement of family was dependent on their own initiative. The typical roles that were fulfilled included accompanying their relative with SCI at all times, helping in basic care, participating as students in the health education sessions, and especially providing emotional support to their family member.

Table 2 Categories and topics emerging from the study

\begin{tabular}{|c|c|}
\hline Categories & Emerging topics \\
\hline $\begin{array}{l}\text { Metamorphosis of the caregiver's profile } \\
\text { This category fundamentally contemplates the discourses that refer to } \\
\text { the drastic change incurred by the appearance of the SCI. A family } \\
\text { restructuring occurs, resulting in a change in their roles, resources, } \\
\text { objectives, profession, and purpose in life. From these discourses, a } \\
\text { subcategory called "change of the roles and tasks of the primary } \\
\text { caretaker" has emerged. From this subcategory, we were interested in } \\
\text { deepening the feelings generated by the new role of the caregiver, the } \\
\text { degree of involvement that depended on the relationship between the } \\
\text { person with SCI and their caregiver, the factors that contribute to the } \\
\text { change in the dynamics of their relationship, and whether their health } \\
\text { status changed after occupying this new role }\end{array}$ & $\begin{array}{l}\text { Change in the roles and tasks of the } \\
\text { primary caregiver }\end{array}$ \\
\hline $\begin{array}{l}\text { Needs } \\
\text { This category highlights the needs of the caregivers during the } \\
\text { rehabilitation period. In this dimension, we analyzed the gaps faced } \\
\text { by the caregivers to offer a holistic approach and obtain a } \\
\text { comprehensive vision from the perspective of the primary caregivers } \\
\text { and the experts in SCI }\end{array}$ & $\begin{array}{l}\text { Social support } \\
\text { Economic support } \\
\text { Psychological support } \\
\text { Mutual aid groups } \\
\text { Information needs } \\
\text { - Evolution of information needs } \\
\text { - Sources of information } \\
\text { Need for care training } \\
\text { - Conditioning factors for caregiver } \\
\text { of person with SCI } \\
\text { - Evolution of information needs } \\
\text { - Stages } \\
\text { - Methodology used for training }\end{array}$ \\
\hline
\end{tabular}


Table 3 Sociodemographic data of the participants

\begin{tabular}{|c|c|c|c|c|c|c|}
\hline \multicolumn{5}{|l|}{ Family caregivers } & \multicolumn{2}{|c|}{ People with SCI } \\
\hline Code & Gender & Socioeconomic & status & Previous caregiving experience & Gender & Injury level \\
\hline Mother of individual injured 10 years ago & $\mathrm{F}$ & Medium & & Yes & M & C7 complete \\
\hline Mother of individual injured 15 years ago & $\mathrm{F}$ & Medium-low & & Yes & $\mathrm{F}$ & D12 incomplete \\
\hline Mother of individual injured 11 years ago & $\mathrm{F}$ & Medium-high & & Yes & $\mathrm{F}$ & C6 complete \\
\hline Husband of individual injured 30 years ago & M & Medium & & Yes & $\mathrm{F}$ & D10 incomplete \\
\hline Wife of individual injured 12 months ago & $\mathrm{F}$ & Medium & & Yes & M & C5 complete \\
\hline Wife of individual injured 11 months ago & $\mathrm{F}$ & Medium-high & & Yes & M & D2 incomplete \\
\hline Wife of individual injured 7 months ago & $\mathrm{F}$ & Medium & & No & M & C6 complete \\
\hline Mother of individual injured 12 months ago & $\mathrm{F}$ & Medium-high & & No & M & C4 incomplete \\
\hline Wife of individual injured 11 months ago & $\mathrm{F}$ & Medium & & No & M & D2 complete \\
\hline Mother of individual injured 4 months ago & $\mathrm{F}$ & Medium & & No & M & D7 complete \\
\hline Wife of individual injured 9 months ago & $\mathrm{F}$ & Medium & & No & M & C5 incomplete \\
\hline Wife of individual injured 6 months ago & $\mathrm{F}$ & Medium & & No & M & C7 complete \\
\hline Daughter of individual injured 3 months ago & $\mathrm{F}$ & Medium & & No & $\mathrm{F}$ & D10 complete \\
\hline Daughter of individual injured 2 months ago & $\mathrm{F}$ & Medium & & No & M & C7 incomplete \\
\hline Wife of individual injured 2 months ago & $\mathrm{F}$ & Medium-low & & No & M & C7 incomplete \\
\hline Wife of individual injured 12 months ago & $\mathrm{F}$ & Medium & & No & M & C6 complete \\
\hline \multicolumn{7}{|l|}{ Professional expert } \\
\hline \multicolumn{2}{|l|}{ Code } & Gender & \multicolumn{3}{|l|}{ Unit } & Hospital \\
\hline Nurse with 6 years of experience & \multicolumn{2}{|c|}{$\mathrm{F}$} & \multicolumn{3}{|c|}{ Neurosurgery } & NHP \\
\hline Nurse with 3 years of experience & \multicolumn{2}{|c|}{$\mathrm{F}$} & \multicolumn{3}{|c|}{ Neurosurgery } & NHP \\
\hline Nurse with 23 years of experience & \multicolumn{2}{|c|}{$\mathrm{F}$} & \multicolumn{3}{|c|}{ Unit with tetraplegic patients } & NHP \\
\hline Nurse with 30 years of experience & \multicolumn{2}{|c|}{$\mathrm{F}$} & \multicolumn{3}{|c|}{ Superintendent of unit with tetraplegic patients } & NHP \\
\hline Nurse with 15 years of experience & \multicolumn{2}{|c|}{$\mathrm{F}$} & \multicolumn{3}{|c|}{ Unit with tetraplegic patients } & NHP \\
\hline Psychologist with 25 years of experience & \multicolumn{2}{|c|}{$\mathrm{F}$} & \multicolumn{3}{|c|}{ Mental health } & NHP \\
\hline Physiotherapist with 6 years of experience & \multicolumn{2}{|c|}{$\mathrm{F}$} & \multicolumn{3}{|c|}{ Rehabilitation } & NHP \\
\hline Social worker with 11 years of experience & \multicolumn{2}{|c|}{$\mathrm{F}$} & \multicolumn{3}{|c|}{ Social worker } & NHP \\
\hline Doctor with 15 years of experience & \multicolumn{2}{|c|}{ M } & \multicolumn{3}{|c|}{ Rehabilitation } & NHP \\
\hline
\end{tabular}

The main caregiver is usually one individual who takes on most of the responsibility, and thus takes on the greatest physical and emotional burden of the care. Of the 25 participants interviewed (Table 3), 90\% were women. The mean age of the primary caregivers was 56.8 years and that of the expert professionals was 47.8 years. Of the 16 family caregivers, at the time of the interview, 10 were in the rehabilitation phase and the other 6 already had been discharged to their homes. Sixty-nine percent of the caregivers were in an average socioeconomic level, $19 \%$ in a mediumhigh level, and $12 \%$ stated they were in a medium-low level. Six were employed at the time of the study, six were unemployed, and four were retired.

The sample of professional health workers included six nurses, one psychologist, one physiotherapist, one social worker, and one rehabilitation physician. The average time of experience with people who had a spinal cord injury was 14 years.

\section{Caregiver metamorphosis}

Before the injury occurred, the caregivers perceived themselves as normal people living normal lives. In their discourses, they alluded to the moment their lives changed: "Due to the accident $[\ldots]$ what a shock...life changed all of us" (Mother of individual injured 10 years ago). Most of the caregivers did not know what a SCI was, its consequences, and their role in this process as caregivers. From this moment on, the period of metamorphosis began.

The caregivers in this study did not have previous experience in care at the time the SCI occurred. Thus, assuming and adjusting to the caregiver role was a difficult journey for them. The analysis of the adjustment phase of the caregiver role allowed us to describe stages, patterns, and trends (Table 4).

Various stages were identified during assumption of the caregiver role. The first stage ( $0-2$ months) consisted of an 
Table 4 Metamorphosis of the caregivers of people with a spinal cord injury

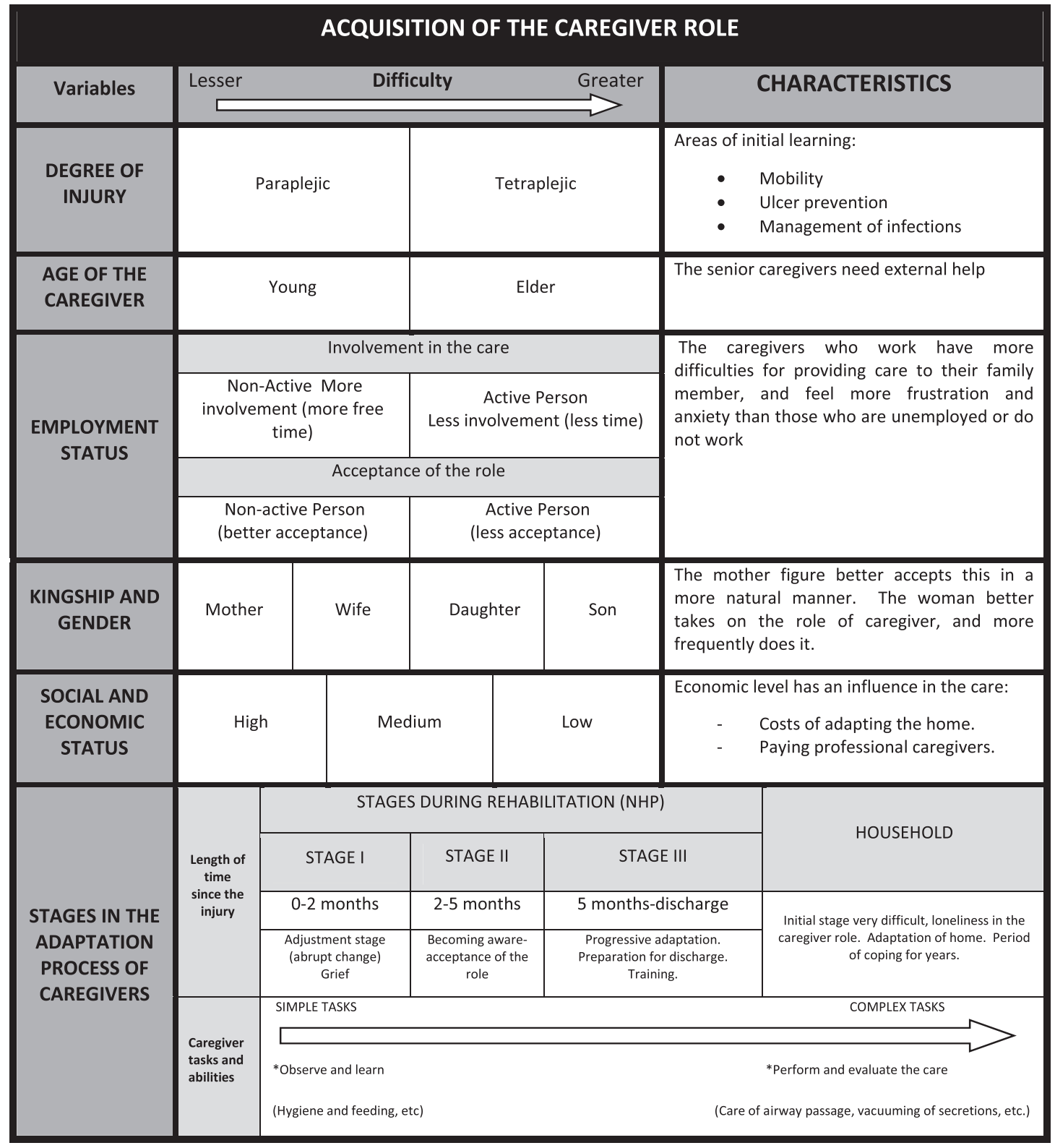

adjustment phase, with the appearance of fear, anguish, worry, uncertainty about whether or not they will be able to take care of everything related to a SCI and its management. The following stage (3-5 months) was characterized by the caregivers becoming progressively conscious of their new responsibility and their limitations due to their inexperience; they started accepting their new role. In this phase, the caregivers went through a period of observation, seeing how the professional workers worked, thus making them participants little by little in basic tasks such as hygiene, feeding, or dressing their family member with SCI, to later, in a progressive manner, introducing more advanced skills such as care of an artificial airway. In this phase, the caregiver started to feel greater strength and capacity for embracing this new role. The professional health workers agreed on the premise that new caregivers should not start with difficult tasks, as they can be shocked by how much work it entails. Lastly, the final stage (5 months until discharge), the caregiver experienced a period of crisis as the date of discharge neared. In this phase, the medical professionals identified the specific needs of the person with SCI and prepared the caregiver for discharge (individualized training on aspects of care, orientation on the adaptation of the home, physical, and 
economic resources). In this phase, fear, worry, and insecurity once again appeared.

In first place, the ease or difficulty in taking on the role of caregiver was related to the severity of SCI. The caregivers of people with tetraplegia, who have greater dependence, showed more difficulties when providing care than caregivers of those with paraplegia. Overall, the former group worried about mobility, the appearance of ulcers due to pressure and infections, and their ability to provide care. Also, the age of the caregiver had an influence on being able to provide care, especially from the physical point of view.

The main caregivers in our studies were in different stages in their personal lives. The caregivers who were actively working $(N=6)$ had feelings of frustration and impotence for not being able to accompany the family member in the rehabilitation process, and thus not being able to become involved in the care. As the caregivers mentioned, combining working life with the caregiver role was very complicated. For the caregivers who were retired, homemakers or unemployed who had more time available, the acceptance, observation, and involvement in the care phase was more fluid, so that they had less anxiety.

Acquisition of the caregiver role is strongly related to the kinship between the caregiver and the person with SCI. Usually, the caregiver role is taken on by women. When the caregiver is the mother, the role is developed in a more natural manner, and the phase of acceptance and initiation of the process is earlier than when the caregivers are wives. Some female caregivers in this study mentioned that helping their husbands during their basic day-to-day care led them to change their role of wives to playing a more maternal role, as they made comparisons to the similarities of caring for a baby, which led them to develop an instinct of protection and responsibility.

"I helped him put his pants on, to hook the crane, to sit him. Although this is a bad comparison, it was just like when you have a baby" (Wife of individual injured 11 months ago).

In this transformation into caregiver, a series of difficulties were found, among which were having to stay strong to provide emotional support to the family member. On the one hand, one of the greatest challenges experienced by the caregivers was the fear of performing complex techniques such as managing the urinary catheter or suctioning secretions from an artificial airway passage. The caregivers externalized a series of needs that appeared during the rehabilitation process (these are described in more detail in the next section): receiving psychological and emotional support, needing to make an economic contribution, and receiving information and training on everything related to the SCI.
The caregiver's metamorphosis was a complex process with both limiting and facilitating elements. The facilitating elements that made the metamorphosis process easier included having direct communication with the professional medical team, having a structured family available to provide emotional support, having economic resources to be able to stay near the hospital during rehabilitation, and receiving psychological support. Also, one of the main pillars the caregivers mentioned during the rehabilitation stage was the contact with other caregivers, the sharing of experiences, and being able to empathize with their peers.

The discourses of caregivers of people with SCI who had been discharged to their homes were different from those who were at the hospital in the rehabilitation phase. The former had gone through a process of progressive coping throughout the years. From these stories, it was noted that the hardest phase was the first 2 months after hospital discharge, during which time they had to adapt to their new situation, adjust their lifestyle, hobbies, work, and so on. They had to eliminate architectural barriers in their homes, and provide care without help from medical professionals who are experts in SCI. The first 6 months included the greatest degrees of stress and anxiety, which lessened with time. The informants mentioned that in order to avoid having the sense of losing themselves, the needed free time, decreasing the number of hours used for providing care, having a good relationship with the person being cared for, receiving external help from a third person, and having economic resources.

Having high socioeconomic status had a positive influence in taking on the role of caregiver, as SCI created a significant economic cost for the family for adapting the home and for receiving help from another person.

The metamorphosis toward the role of caregiver is a personal and family-oriented process. In some cases, metamorphosis patterns with resignation are found, and in other cases, with resilience. Caregivers who have reached a greater personal and family-based adaptation to their new lives have shown some factors leading to overcoming the situation such as verifying that their family is physically, socially, and psychologically fit, living in the present, having high economic support that allows them to be less preoccupied with work and accepting their loved one's injury. Some caregivers become stronger after the fact, learn from the traumatic experience, and believe that the SCI has changed the course of their lives. In the following testimonies, we can see these insights:

"Mom, why did this have to happen to me? And I said: I don't know. I can only tell you that it will bring something better. And later I have verified it. I have seen how his life has changed, how things have been granted to him, how he has grown as a person and was 
pushed aside from a turbulent adolescence" (Mother of individual injured 10 years ago).

\section{Caregiver needs}

\section{Social support}

The emotional support provided by the social environment becomes indispensable for caregivers. The discourses revealed that they rely on family and friends; God, on a spiritual level; or themselves, on an individual level.

"In God and my family" (Mother of individual injured 11 years ago).

"It's just that you have surmount all the challenges that life throws at you. Faith in God has helped me a lot" (Mother of individual injured 4 month ago).

However, the support that caregivers receive from their social networks decreases over time. What at first were continuous visits become more infrequent, with only good friends remaining at the end.

Another fundamental pillar of support is the caregivers themselves. During the hospitalization and rehabilitation process, mutual aid groups are created where the caregivers can express their concerns, doubts, and fears. The person with SCI can have a series of multidisciplinary activities planned within the rehabilitation program throughout the day as well as periods of rest stipulated by the hospital protocol; thus, strict visitor schedules are enforced, so many hours exist between corridors when affective bonds are ultimately established.

"However, you always have doubts, fears.... Here, we have met other caregivers who help each other" (Wife of individual injured 2 month ago).

Caregivers occasionally seek understanding, words of comfort, and a shoulder to cry on.

\section{Economic support}

Many caregivers must seek accommodation to accompany their family member throughout the rehabilitation process, which implies a significant financial outlay for the primary caregiver.

Most of discourses portrayed institutional support as unfair. Criticism was directed at the poor distribution of economic resources and scarce subsidies. A NHP social worker stated:
"Moving forward requires a significant financial outlay. All of the basic technical aids, such as a crane for a tetraplegic, are significant expenses that can't always be acquired out of dependency (funds). A crane can cost you $€ 6,000$ or $€ 7,000$ " (Mother of individual injured 10 years ago).

An additional problem is the need to adapt a home (e.g., some participants lived in a home without an elevator). The costs that the person with SCI and his or her family must assume are great.

\section{Psychological support}

Upon admission to the National Paraplegic Hospital, a psychological care plan was established in which both the patient with SCI and the family were involved. The coping strategies that the family initially had and how they overcame difficult situations in the past were evaluated.

A psychologist from the mental health team stated that it is occasionally difficult to reach the person to be able to help:

"It is true that there are people who need to contain the situation during the first stage when they arrive as they are so affected.... There are those who do not leave and remain there until the end. There are those who do the first part and leave it. There are those who do not come at first because they cannot hear more things they do not want to hear anymore, and then there are those who come when the final stage has already begun" (Nurse with 6 years of experience).

The psychologist revealed that when a person finds him or herself in a state of dependence, such as tetraplegia, and the person affected is a child, overprotection by the parents is almost automatic. In the following quote, we can see how the caregiver is conscious of this protection, but cannot avoid it.

"You have to dissociate yourself a bit, but it's just that I don't want anything to happen to her. I know that I overprotect her, and this tires her. But I suffer..." (Mother of individual injured 10 years ago).

At first, most of the primary caregivers rejected the psychological help provided. As they moved forward in the rehabilitation phase, the moment arrived when they perceived the psychological support received as being positive and considered it necessary and essential that a program of psychological care be established for patients with SCI and their families. 


\section{Need for information}

Caregivers must be informed at all times on the health and progress of their relatives. During the critical or acute period, the health professionals might provide misinformation as in the following discourse:

"I did not even know what injury he had. In fact when we got the first medical report... it was then that I read that he had a D2. You assume... all assumptions... that if you go to a paraplegic hospital it is because he is paraplegic; but in truth, we did not know anything" (Wife of individual injured 11 month ago).

Confrontations occur between relatives and health professionals with regard to when and how to introduce the information. The professionals reported that the approach should be made slowly and progressively without overwhelming the recipient; however, the caregivers referred to a certain degree of stress from not knowing most of the necessary information from the beginning in their discourses.

A proper schedule for the release of information or a communication protocol for health professionals did not exist; therefore, the circumstances, emotional state, and whether participants requested to be informed were determining factors.

More information was gleaned from the health professionals after attending talks, searching the Internet, joining associations, and attending courses.

\section{Need for training}

The primary caregivers must learn and be trained with regard to all of the individualized care related to SCI, both theoretically and practically. According to SCI experts, important conditioning factors should be considered when starting training with caregivers. First, identifying the primary caregiver is indispensable; this task was often difficult as this figure was not clearly determined until some time had passed. Other factors are age, cognitive and cultural education levels, the environment, and fears. In addition, the professionals stated that the emotional instability of the family caregiver was a limiting factor in the teachinglearning process of caregiving.

The professionals interviewed were unclear about the ideal time to start training, although they agreed that it depended on the emotional states of the primary caregiver and the patient.

Caregivers go through a series of phases from the beginning of care until they master the competencies required to take care of their family member. During the first phase, they must accept their role and feel prepared to collaborate with nursing professionals. There are negative feelings in the face of uncertainty regarding whether they will be able to perform all of the care that a person with SCI needs. This first phase is the most difficult stage for caregivers.

The second stage consists of the "training stage." During this phase, the caregivers proceed through a period of observation, watching how the professionals work, gradually participating in basic tasks such as hygiene, feeding, and dressing. Later, they progressively are introduced to more advanced skills such as caring for an artificial airway.

Finally, acquiring caregiving skills entails repetitive training on specific tasks. Before discharge, the professionals ensure that caregivers have all of the care competencies, although some testimonies revealed that the caregivers felt some areas were not trained or enforced enough.

"I (only) used the resuscitation bag once... Then, the next time I will not remember if you have to remove the plug, the cannula, and you if you have to plug the other thing in...it is not something that I feel confident about." (Wife of individual injured 6 month ago).

\section{Discussion}

Patients with SCI and their primary caregivers undergo a metamorphosis in every sphere of their lives. In Spain, patients are discharged to their homes after a rehabilitation period, which for our sample ranged between 6 and 12 months. The caregiver role was a differentiating element compared to other countries in our area, where the patients are institutionalized. Therefore, the study was conducted to analyze the role of the caregiver in our context.

We were not able to determine a unique pattern of metamorphosis that was more common. In general, as shown in Table 4, we found a great number of variables that specifically described each case. However, a more general pattern of metamorphosis was found, in which better adaptation to the role of caregiver was achieved: mother, not employed, young and with high economic resources. There are currently no previous studies in our cultural context that could be used to compare our results.

The metamorphosis of the caregiver is a process in which the caregiver evolves along different stages. In this study, three phases were identified that represent the manner in which family caregivers faced the processes of change and coping (which have been defined as metamorphosis of the caregiver). The stages are: (1) adjustment-mourning stage, (2) acceptance of the caregiver role stage, and (3) adaptation-preparation for discharge stage. These stages are 
similar to other models described in the bibliography on family adaptations to traumatic events, such as the Family Adjustment and Adaptation Responses (FAAR) model [12]. As this model states, the families of those having a SCI go through cycles of adjustment, crisis, and adaptation. They did not always make progress in a direct and linear manner through the processes experienced during the pre-crisis (before the accident or traumatic even occurred), crisis (during the acute phase of the SCI), and post-crisis (once there is an acceptance of the situation) periods.

In our sample, the responsibility of the role of caregiver was normally given to a single person in the family. Likewise, and through the analysis of the caregiver model within the framework proposed by Gray-Davidson [13], we cannot attest that our informants belonged to a single caregiving model, but they answered to diverse stereotypes, as there were some that were solitary caregivers, others answered to collaborative model (family working together), although most could belong to the observed caregiving model (one family member is responsible, the others voice their opinions). In our study, a caregiver in the initial adjustment phase corresponded to a collaborative model, as many members of the family collaborated and supported each other. The next model was identified with an observation model, as a unique main caregiver was defined as the one responsible for the care. In the last stage, a caregiver assumed most of the responsibility, sometimes showing feelings of loneliness, especially when the patient was discharged home.

As described, there were some patterns in the metamorphosis of the caregiver, but a series of facilitating factors also were identified, which could diminish or eliminate negative feelings in the caregivers such as receiving emotional and psychological support, having more communication with the team of professionals to receive positive feedback for the work that they performed, and having economic and social resources. Our results are similar to those from a study conducted in USA [4], which identified both positive and negative factors when taking on the role of caregiver. As in our study, among the positive aspects, it was highlighted that the caregiver tends to experience personal growth, as well as improvement in family relationships.

On the other hand, in their discourses, some caregivers mentioned states of anxiety and depression associated with the role they play. In this sense, several studies have shown that a high percentage of caregivers of people with SCI develop depression [14, 15], and this development depends on the caregivers' abilities to resolve conflicts and their capacity for psychological adjustment [16]. Other authors have concluded that successful functioning with the family, providing coping skills, and receiving social and economic support promoted positive results and effectively reduced caregiver burden [17-19]. Thus, the team of professionals has a fundamental role during the rehabilitation stage, assessing the caregiver in a holistic and individualized manner in order to provide appropriate resources and thus helping reduce caregiver burden.

The most important needs detected by the caregivers were psychological support, social support, economic resources, information and training throughout the treatment process, and the creation of informal mutual aid groups. Although important cultural differences can exist, these needs coincide with the results of a previous studies $[4,6$, 20].

It is essential for primary caregivers to have a social support network, primarily composed of family, friends, professional health workers, and the caregivers themselves. A recent study revealed that the relationship between social support and caregiver coping positively affected their quality of life and helped in avoiding fatigue, burnout, and psycho-emotional exhaustion [21]. Consistent with this work, Elliot and colleagues [14] showed that the ability to be positive as well as social support networks helped caregivers to be resilient. Positive emotions can facilitate adjustment in times of stress because they promote flexibility and the ability to attend to and integrate new information [22, 23]. Social support is one of the factors that mitigates the caregiver burden from SCI [24]. The participants interviewed at their homes identified receiving help from a third person as a primordial need, especially when it involved caring for a person with tetraplegia. The additional support of hired caregivers could be an option for some; however, a few family caregivers showed dissatisfaction with the services of the hired help due to elevated costs and the lack of training. This created more frustration than satisfaction, so this idea was often abandoned. Another fundamental pillar within the need to receive social support is that caregivers need to share their feelings. Previous studies $[5,10,25]$ have indicated that creating spaces to form informal support networks for mutual information engenders benefits both for the individual with SCI and for the family.

In the rehabilitation stage, caregivers can share and empathize with other caregivers that find themselves in the same situation. However, when at home, they feel emptiness and loneliness. It would be useful to create spaces where caregivers of people with SCI could learn and share their situation, to clarify ideas and to freely express their concerns and troubles.

Economic resources are also essential for people with SCI and their families in order to achieve a high quality of life $[8,20]$. Our participants considered many of the sociohealth services as essential, but these services were not included in free public coverage. One study stated that economic factors were the major barriers for accessing 
appropriate services and healthcare resources, directly influencing patient quality of life and, often, the health of the family caregiver [26].

In Spain, the social security services offer economic resources depending on the level of yearly income of each individual and the degree of disability. Most of the informants mentioned that these subsidies were insufficient for cover their needs (adaptation of the home, health resources, support for the caregiver, crane, wheelchair), but more than money, it was more important for them to maintain a stable house and solid emotional levels.

In relation to the need to receive psychological support, during the rehabilitation stage, most caregivers positively valued receiving psychological therapy; however, some participants flatly refused this. In a recent study conducted in Spain on stigma and mental health, there was a large percentage of people who were affected by mental health problems that anticipated the appearance of stigmatizing attitudes [27]. They believed they would be undervalued and discriminated against due to suffering from a mental disorder, such as anxiety or depression, and the fact of receiving psychological therapy. It could be that Spanish culture is reticent with regard to receiving psychological treatment (because mental illness remains taboo). Psychological interventions and teaching problem-solving skills are essential for caregivers of people with SCI $[13,14]$.

Caregivers obtained most of their information from expert practitioners; however, they claimed they were not informed enough in some cases. Previous studies also have supported this claim [10, 24]. In contrast, professionals believed that information should be issued gradually, so that the concepts are assimilated, thereby avoiding trauma. There was no structured schedule for releasing information, nor did a communication protocol for health professionals exist; therefore, the circumstances, emotional state, and whether participants request to be informed were determinants [26].

Consistent with our results, numerous studies $[24,28$, 29] have indicated that caregivers require more education and training regarding care. Initiating caregiver training is difficult because many relatives remain stuck in the denial phase; therefore, they avoid all types of information and training. Emotions considered as negative impede the assimilation of concepts and block cognitive abilities [14]. Besides the emotional component, we identified other factors that affect training such as the age of the caregiver, cultural education level, fear, and the frustration and context in which the training occurs. These factors should be taken into account during the training phase of the caregivers. Based on the results of the study, we can attest that the systems of support are very important for helping family caregivers meet their needs and overcome challenges. A few caregivers do not adapt well to their new situation, creating an environment of discomfort and getting the impression of bad quality. It is up to professionals who work in the field of rehabilitation to give information to the caregivers and to detect family, social, belief and interpersonal relations support systems, so that they can control the possible limiting/stressor elements and can bolster the facilitating elements in order to ensure that the caregivers become more resilient [30, 31].

There were differences found between caregivers who had more than a year of caregiving experience and those who were still the rehabilitation phase. The caregivers who were still in the critical phase and had not been discharged yet showed signs or worry, anxiety and fear of the unknown. However, in the group of chronic caregivers, some of them had resigned themselves while others were resilient. This last group thought of themselves as having a good quality of life, and had a great support network. A study conducted in the Netherlands [32] showed that a great number of caregivers can become resilient after the first year after the SCI. The key was the early detection and development of psychological interventions for people who had difficulties with posttraumatic growth, as it was unlikely that their discomfort could be reduced without treatment in a year. There are studies on the resilience of caregivers after a year post SCI, so our results cannot compared.

For caregivers who were in the first phase at the NHP, there were more negative aspects reported, such as fatigue, fear, worry, and change in family dynamics. For those who had more than a year of experience as caregivers of a person with SCI, we found an important change in their statements, with more positive items such as greater family cohesion and personal growth. This change in the equilibrium of positive aspects and negative ones has also been described in another study, although a comparison with the caregiving length of time was not performed [4]. Also, our results were in agreement with a systematic review of caregivers to people with SCI [33], which described how caregivers identified sources of strength that helped them to manage stress, including faith and support from friends and coworkers.

Metamorphosis of the caregiver is a complex and dynamic process conditioned by multiple elements and circumstances. This study showed how a series of interventions (including family training, training in conflict resolution, information on all the available resources, and support groups) provided benefits for caregivers, allowing for a greater and better adaptation of the caregiver role. These effects were even more relevant after discharge to home. It is up to the professionals in rehabilitation centers to include the role of the caregiver as an integral part of the patient's rehabilitation.

Future research should focus on the creation of a validated scale to identify needs of family caregivers, following 
all the corresponding validation procedures in this cultural context. Lastly, the need to establish a formal training and support program that involves the caregiver in the rehabilitation of a person with SCI is indispensable.

\section{Limitations}

The results of the study could only be partially generalizable, due to the sample size of the study and the cultural, social, and healthcare characteristics of our context.

\section{Implications}

This study highlights the role of the caregiver and reveals the need to allocate different types of resources (information, psychological and social support, training, and so on), taking into account the needs of caregivers of people with SCI. Also, it underscores the importance of early detection and the development of psychological interventions for people who have difficulty with post-traumatic growth. Moreover, the structured evaluation of the needs of the family caregivers of people with SCI is needed during the different stages of the process.

Acknowledgements This study would not have been possible without the close collaboration of the National Hospital for Paraplegics in Toledo. We thank them for their support, and we thank the excellent staff of health professionals who work every day to improve the quality of life of patients with SCI and their families. We thank the association for the disabled, ASPAYM, and their participation in the project. They opened the doors of their association to allow us to interview the relatives of people with SCI. We also thank the NGO "Messengers of Peace" who helped with the participant selection for interviews. This study was supported by a research project awarded by the Catholic University of Murcia (PMAFI 25/14). The project was endowed with a €4891 grant.

\section{Compliance with ethical standards}

Conflict of interest The authors declare that they have no conflict of interest.

\section{References}

1. Kemp LA. Care and services for spinal injured people with, and without, neurological deficit. Disabil Rehabil. 2002;24:810-6.

2. Dickson A, O'Brien G, Ward R, Allan D, O'Carroll R. The impact of assuming the primary caregiver role following traumatic spinal cord injury: an interpretative phenomenological analysis of the spouse's experience. Psychol Health. 2010;25:1101-20.

3. Gajraj-Singh P. Psychological impact and the burden of caregiving for persons with spinal cord injury (SCI) living in the community in Fiji. Spinal Cord. 2011;49:928-34.

4. Charlifue SB, Botticello A, Kolakowsky-Hayner SA, Richards JS, Tulsky DS. Family caregivers of individuals with spinal cord injury: exploring the stresses and benefits. Spinal Cord. 2016;54:732-6.

5. Dickson A, Ward R, O'Brien G, Allan D, O'Carroll R. Difficulties adjusting to post-discharge life following a spinal cord injury: an interpretative phenomenological analysis. Psychol Health Med. 2011;16:463-74.

6. Foster M, Amsters D, Carlson G. Spinal cord injury and family caregivers: a description of care and perception of service need. Aust J Prim Health. 2005;11:91-101.

7. Post MW, Bloemen J, De Witte LP. Burden of support for partners of persons with spinal cord injuries. Spinal Cord. 2005;43:311-9.

8. Morrison M, Kelly EH, Russell HF, Vogel LC. Rewards of parenting children and adolescents with spinal cord injuries. Spinal Cord. 2017;55:52-8

9. Nogueira PC, Rabeh SA, Caliri MH, Dantas RA, Haas VJ. Burden of care and its impact on health-related quality of life of caregivers of individuals with spinal cord injury. Rev Lat Am Enferm. 2012;20:1048-56.

10. Conti A, Garrino L, Montanari P, Dimonte V. Informal caregivers' needs on discharge from the spinal cord unit: analysis of perceptions and lived experiences. Disabil Rehabil. 2016;38:159-67.

11. Colaizzi PF. Psychological research as the phenomenologist views it. In: Valle R, King M, editors.Existential phenomenological alternatives for psychology. Oxford: Oxford University Press; 1978. p. $48-71$.

12. Patterson JM. Families experiencing stress: I. The family adjustment and adaptation response model: II. Applying the FAAR model to health-related issues for intervention and research. Fam Syst Med. 1988;6:202-37.

13. Davidson FG. The Alzheimer's sourcebook for caregivers: a practical guide for getting through the day. McGraw-Hill/Contemporary; Illinois (U.S.A.) 1996.

14. Elliott TR, Berry JW, Richards JS, Shewchuk RM. Resilience in the initial year of caregiving for a family member with a traumatic spinal cord injury. J Consult Clin Psychol. 2014;82:1072-86.

15. Elliott TR, Berry JW. Brief problem-solving training for family caregivers of persons with recent-onset spinal cord injuries: a randomized controlled trial. J Clin Psychol. 2009;65:406-22.

16. Lui MH, Ross FM, Thompson DR. Supporting family caregivers in stroke care. Stroke. 2005;36:2514-22.

17. Adelman RD, Tmanova LL, Delgado D, Dion S, Lachs MS. Caregiver burden: a clinical review. JAMA. 2014;311:1052-60.

18. Baker A, Barker S, Sampson A, Martin C. Caregiver outcomes and interventions: a systematic scoping review of the traumatic brain injury and spinal cord injury literature. Clin Rehabil. 2017;31:45-60.

19. Secinti E, Yavuz HM, Selcuk B. Feelings of burden among family caregivers of people with spinal cord injury in Turkey. Spinal Cord. 2017;55:1-6.

20. Arango-Lasprilla JC, Nicholls E, Olivera SL, Perdomo JL, Arango JA. Health-related quality of life in individuals with spinal cord injury in Colombia, South America. NeuroRehabilitation. 2010;27:313-9.

21. Rodakowski J, Skidmore ER, Rogers JC, Schulz R. Role of social support in predicting caregiver burden. Arch Phys Med Rehabil. 2012;93:2229-36.

22. Cohn MA, Fredrickson BL, Brown SL, Mikels JA, Conway AM. Happiness unpacked: positive emotions increase life satisfaction by building resilience. Emotion. 2009;9:361-8.

23. Fredrickson BL. Positive emotions broaden and build. Adv Exp Soc Psychol. 2013;47:53

24. Lucke KT, Martinez H, Mendez TB, Arévalo-Flechas LC. Resolving to go forward: the experience of Latino/Hispanic family caregivers. Qual Health Res. 2013;23:218-30. 
25. Sheija A, Manigandan C. Efficacy of support groups for spouses of patients with spinal cord injury and its impact on their quality of life. Int J Rehabil Res. 2005;28:379-83.

26. Kim HR, Shin HI. When is it appropriate to deliver a prognosis to Korean persons with acute spinal cord injury? Arch Phys Med Rehabil. 2012;34:1396-403.

27. Brea J, Gil H. Estigma y salud mental. Una reflexión desde el trabajo social. Trabajo Social Hoy. 2016;78:95-112.

28. Manns PJ, May La. Perceptions of issues associated with the maintenance and improvement of long-term health in people with SCI. Spinal Cord. 2007;45:411-9.

29. Smith EM, Boucher N, Miller WC. Caregiving services in spinal cord injury: a systematic review of the literature. Spinal Cord. 2016;54:562-9.
30. Rahmani Rasa A, Hosseini SA, Haghgoo H, Khankeh HR, Ray G. Review paper: aspects related to resilience in people with spinal cord injury. Arch Rehabil. 2017;17:350-9.

31. Chen H, Boore J. Living with a relative who has a spinal cord injury: a grounded theory approach. J Clin Nurs. 2009;18:174-82.

32. Elliot RS, Berry JW, Scott JR, Shewchuk RM. Resilience in the initial year of caregiving for a family member with a traumatic spinal cord injury. J Am Psychol Assoc. 2014;82:1072-86.

33. Lynch J, Cahalan R. The impact of spinal cord injury on the quality of life of primary family caregivers: a literature review. Spinal Cord. 2017;55:1-15. 